\title{
S-adenosyl methionine specifically protects the anticancer effect of 5-FU via DNMTs expression in human A549 lung cancer cells
}

\author{
MYEONG-SUN HAM*, JU-KYUNG LEE* and KEUN-CHEOL KIM \\ Medical and Bio-Material Research Center and Department of Biological Sciences, College of Natural Sciences, \\ Kangwon National University, Chuncheon, Kangwon 200-701, Republic of Korea
}

Received June 11, 2012; Accepted December 11, 2012

DOI: $10.3892 / \mathrm{mco} .2012 .53$

\begin{abstract}
Cellular methylation is associated with stabilization of the chromatin structure. S-adenosyl methionine (SAM), a metabolite of methionine metabolism, is the methyl donor of essential cellular methyltransferase reactions. Using 3-(4,5-dimethylthiazol-2-yl)-2,5-dephenyl tetrazolium bromide (MTT) assay, we found that combination treatment of SAM and 5-fluorouracil (5-FU) specifically protected the anticancer effect of 5-FU, whereas the combination of SAM and cisplatin had no effect. This result was confirmed by FACS analysis. The combination treatment of SAM and 5-FU significantly decreased the dead cell population, while the G1 cell population was slightly increased, suggesting that protection of SAM is not associated with the cell cycle arrest of DNA-damaging drugs. We also analyzed which cellular methylation-related proteins were involved in the protective effect. Results showed the expression of DNA methyltransferases (DNMTs) was decreased with 5-FU alone but was increased with the combination treatment of SAM and 5-FU, suggesting that SAM protects the anticancer effect of 5-FU by regulating the expression of DNMTs. Taken together, the results indicated that SAM specifically modulates the anticancer effect of the DNA damage agent 5-FU and this may be modulated by aberrant DNA methylation.
\end{abstract}

\section{Introduction}

DNA-damaging drugs induce death in malignant tumor cells. Although the majority of these drugs affect the primary structure of DNA, their usefulness may be limited by the chromatin

Correspondence to: Dr Keun-Cheol Kim, Department of Biological Sciences, College of Natural Sciences, Kangwon National University, 1 Kangwondaehak Road, Chuncheon, Kangwon 200-701, Republic of Korea

E-mail: kckim@kangwon.ac.kr

*Contributed equally

Key words: S-adenosyl methionine, 5-fluorouracil, cisplatin, DNA methyltransferases, protection structure that represents the physiological template of the DNA. Since methylation of DNA or proteins occurs in the nucleus, the anticancer effect of DNA-damaging drugs may be affected by cellular methylation $(1,2)$.

S-adenosyl methionine (SAM) is key as a methyl donor in methylation reactions. SAM transfers the methyl group, $\mathrm{CH}_{3}$, to cell components such as DNA, proteins and lipids $(3,4)$. It is made from adenosine triphosphate and methionine by methionine adenosyltransferase. Removal of the methyl group from SAM yields S-adenosyl homocysteine (SAH), which acts as a methyltransferase inhibitor (5,6). Metabolic SAM can be synthesized throughout the body, but most SAM is consumed in the liver $(7,8)$. DNA methyltransferases (DNMTs) catalyze the transfer of a methyl group from SAM to cytosine residues to form 5-methyl cytosine at $\mathrm{CpG}$ sites in the genome, an important regulatory mechanism for regulation of the gene expression (9). Histone methyltransferases (HMTases) also utilize SAM to methylate lysine or the arginine residue of histone proteins (10). As SAM is a key metabolite of hepatocyte growth, death and differentiation, its use in treatment may improve survival in liver disease $(11,12)$. Recently, multiple clinical trials have also indicated that SAM is important in the treatment of Alzheimer's disease, depression and osteoarthritis $(13,14)$. However, therapeutic usages of SAM are not yet proven in cancer.

5-Fluorouracil (5-FU) has been used in the treatment of various types of cancer (15-17). Since 5-FU acts as a thymidylate synthase inhibitor, it blocks the synthesis of thymidine during DNA replication. However, the correlation between thymidylate synthase levels and 5-FU sensitivity remains controversial, although it is widely thought that thymidylate synthase is the main molecular mechanism governing 5-FU sensitivity (18-20). Other investigators have suggested that 5-FU resistance may also be induced by p53 gene mutation, mismatch repair gene deficiency, deregulation of pyrimidine metabolism-related enzymes and overexpression of anti-apoptotic factors $(19,21)$. Cisplatin is a platinum-based chemotherapy drug that causes DNA crosslinking. Cisplatin is frequently administered as part of a combination chemotherapy regimen with other drugs as it sometimes acts in synergy synergistic with other agents $(22,23)$.

In the present study, we hypothesized that SAM has an impact on the cytotoxic effect of DNA-damaging drugs. We characterized and compared the effects of SAM and 
assessed whether it affects the anticancer effects of 5-FU and cisplatin. Using several cytotoxic assays, we showed that SAM specifically regulates the anticancer effect of 5 -FU but not that of cisplatin.

\section{Materials and methods}

Cell culture and reagents. TheA549 human lung cancer cell line was obtained from ATCC (Baltimore, MD, USA). Cells were maintained in DMEM medium supplemented with $10 \%(\mathrm{v} / \mathrm{v})$ fetal bovine serum, and penicillin-streptomycin $(100 \mathrm{U} / \mathrm{ml})$ at $37^{\circ} \mathrm{C}$ in a humidified incubator containing $5 \%$ $\mathrm{CO}_{2}$. SAM, 5-FU and cisplatin were purchased from Sigma (St. Louis, MO, USA) and dissolved in dimethyl sulfoxide for further use. Combination treatment of SAM and anticancer drugs was performed as indicated in figure legends.

3-(4,5-dimethylthiazol-2-yl)-2,5-dephenyl tetrazolium bromide (MTT) assay. Various concentrations of 5-FU (2-40 $\mu \mathrm{M})$ or cisplatin $(2-32 \mu \mathrm{M})$ were treated.Cells $(1,000$ cells/ well) were seeded and treated with various concentrations of the indicated drugs for 2 days. MTT (Sigma) was dissolved in phosphate-buffered saline (PBS) and filtered through a $0.2 \mu \mathrm{m}$ filter. The solution was stored at $4^{\circ} \mathrm{C}$ for future use. To evaluate cell viability, A549 cells were seeded in 96-well plates at densities of 500-1000 cells/well. The following day, the cells were treated with the indicated drugs. After washing with PBS, the cells were incubated in MTT solution for $30 \mathrm{~min}$. Absorbance was measured using a microplate reader (Bio-Rad, Hercules, CA, USA) at a wavelength of $540 \mathrm{~nm}$. After the experiment was performed, the mean and standard deviation of the data were calculated. Statistical analysis was performed using the Student's t-test. $\mathrm{P}<0.05$ was considered to indicate a statistically significant difference.

FACS analysis. Cells were seeded in $1 \times 10^{6}$ cells in $100-\mathrm{mm}$ dishes and treated with SAM, 5-FU and cisplatin. The cells were harvested and fixed with $70 \%$ cold ethanol. The fixed cells were then washed with PBS and stained with $50 \mu \mathrm{g} / \mathrm{ml}$ propidium iodide containing $1 \mathrm{mg} / \mathrm{ml}$ R Nase A for $15 \mathrm{~min}$ in a $37^{\circ} \mathrm{C}$ water bath. Analyses of 10,000 events were obtained on a FACSCalibur flow cytometer (Becton-Dickinson, Mountain View, CA, USA) and the cell cycles were analyzed using ModFit DNA analysis software.

Western blot analysis. Cells were cultured in 100-mm dishes and treated with the indicated drugs. The cells were collected by scraping and lysed with RIPA lysis buffer $(0.02 \mathrm{M}$ Tris, $0.15 \mathrm{M}$ $\mathrm{NaCl}, 0.1 \%$ sodium dodecyl sulfate DS, $1 \%$ Triton X-100, $1 \%$ sodium deoxycholate, $0.02 \mathrm{mM}$ phenylmethylsulfonyl fluoride, $0.1 \mathrm{mM} \mathrm{NaF}, 0.01 \mathrm{mg} / \mathrm{ml}$ leupeptin, $0.01 \mathrm{mg} / \mathrm{ml}$ pepstatin) and then centrifuged at $14,200 \mathrm{xg}$ for $30 \mathrm{~min}$ at $4^{\circ} \mathrm{C}$. The amount of protein was determined with the Bradford protein assay (Bio-Rad). The lysates were boiled for $5 \mathrm{~min}$, separated by SDS-polyacrylamide gel electrophoresis and transferred to polyvinylidene difluoride membrane (Amersham Biosciences, Piscataway, NJ, USA). The membranes were incubated for 1 $\mathrm{h}$ with blocking buffer [5\% non-fat milk and $0.1 \%$ Tween-20 in Tris-buffered saline (TBS-T)] and then incubated with the specific antibodies. The membranes were washed three times with TBS-T and incubated for $1 \mathrm{~h}$ with secondary antibodies (Santa Cruz Biotechnology Inc., Santa Cruz, CA, USA). Proteins were detected with enhanced chemiluminescence reagent (Amersham Biosciences).

$R T-P C R$. Cells were collected after treatment with the indicated drugs. Total RNA was extracted using TRIzol-reagent (Promega Co., Madison, WI, USA) according to the manufacturer's instructions. cDNA was synthesized using reverse-transcriptase according to the manufacturer's instructions and was used as the template for PCR amplification. Primer sequences of DNMTs-specific primer sets are as follows: DNMT1, sense: 5'-ATC TAC CAG TGT ACA GAG TGT GA-3', antisense: 5'-ATA CTG ACA GAA GTA ATC TCG AT-3'; DNMT3A, sense: 5'-ATC TAC CAG TGT ACA GAG TGT GA-3', antisense: 5'-ATA CTG ACA GAA GTA ATC TCG AT-3'; DNMT3B, sense: 5'-ATC TAC CAG TGT ACA GAG TGT GA-3', antisense: 5'-ATA CTG ACA GAA GTA ATC TCG AT-3'; GAPDH, sense: 5'-ATG ACA ACT TTG GCA TTG TGG AA-3', GAPDH antisense: 5'-CTG TTG CTG TAG CCG TAT TCA TT-3'. GAPDH was used as a loading control. Each sample was incubated at $95^{\circ} \mathrm{C}$ for $20 \mathrm{sec}, 60^{\circ} \mathrm{C}$ for $25 \mathrm{sec}$ and $72^{\circ} \mathrm{C}$ for $30 \mathrm{sec}$ for 35 cycles. Reaction samples were then incubated for an additional $7 \mathrm{~min}$ at $72^{\circ} \mathrm{C}$ and cooled to $4^{\circ} \mathrm{C}$. PCR products were resolved on $1 \%$ agarose gel.

\section{Results}

SAM-modulates the anticancer effect of 5-FU but not cisplatin. DNA-damaging drugs activate apoptosis in cancer cells. We treated various concentrations of 5-FU $(2-40 \mu \mathrm{M})$ or cisplatin $(2-32 \mu \mathrm{M})$ for $48 \mathrm{~h}$ in A549 lung cancer cells and performed MTT assay to analyze drug sensitivity. As expected, 5-FU or cisplatin resulted in cytotoxic effects in a dose-dependent manner (Fig. 1). $\mathrm{LD}_{50}$ of 5 -FU and cisplatin were $\sim 7$ and $6 \mu \mathrm{M}$, respectively. We also performed MTT assay by treatment using SAM $(5-40 \mu \mathrm{g} / \mathrm{ml})$. SAM treatment produced no special morphological changes and had no distinct cytotoxic effect in these ranges. However, since SAM modulates cellular methylation of DNA or proteins, we hypothesized that SAM affected DNA-damaging drugs. MTT assay was performed at several concentrations of 5-FU and cisplatin in combination with SAM of $20 \mu \mathrm{g} / \mathrm{ml}$. Combination treatment of SAM and 5-FU somewhat protected the anticancer effect of 5-FU. However, this phenomenon was not observed in the combination treatment of SAM and cisplatin (Fig. 2). The protection effect of SAM on 5-FU was evident from 3 days after the combination treatment of SAM and 5-FU. This result suggests that SAM specifically modulates the anticancer effect of 5-FU but not for cisplatin.

Protective effect of SAM on the anticancer effect of 5-FU does not require specific cell cycle arrest. FACS analysis was performed to examine the manner in which SAM affects cell cycle arrest or cell death by 5-FU or cisplatin. Treatment with SAM only did not show any difference as compared with the control. Treatment of 5-FU induced cell death, but not specific cell cycle arrest, whereas cisplatin induced G2/M arrest (Fig. 3A). However, the combination treatment of SAM and 5-FU significantly decreased the dead cell population, 
5-FU

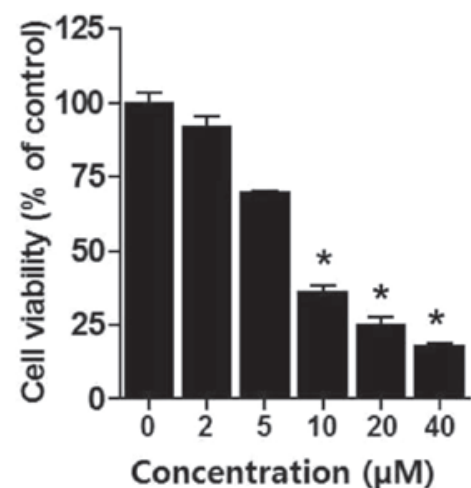

Cisplatin

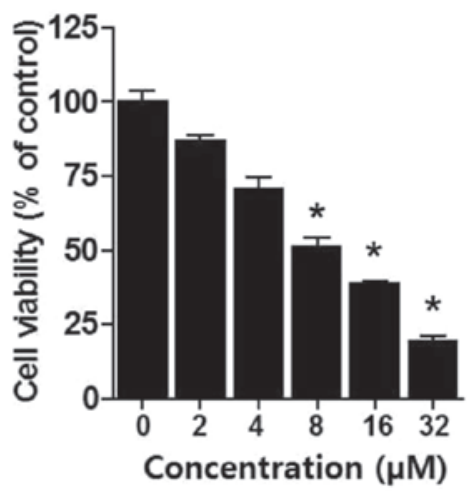

SAM

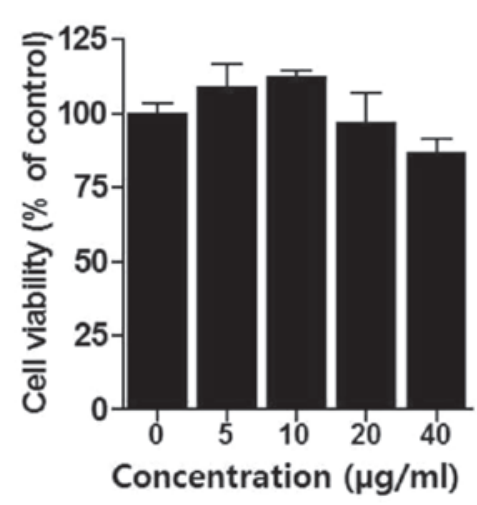

Figure 1. Effect on the cell growth of S-adenosyl methionine (SAM), 5-fluorouracil (5-FU) and cisplatin. Cells (1,000 cells/well) were seeded and treated with various concentrations of the indicated drugs for 2 days and this was followed by 3-(4,5-dimethylthiazol-2-yl)-2,5-dephenyl tetrazolium bromide (MTT) assay. SAM exhibit no specific cytotoxic effect in A549 cells. However, 5-FU and cisplatin significantly reduced cell growth. Data are shown as the mean \pm standard deviation of triplicate assays. ${ }^{*} \mathrm{P}<0.05$, compared to the control group.

A

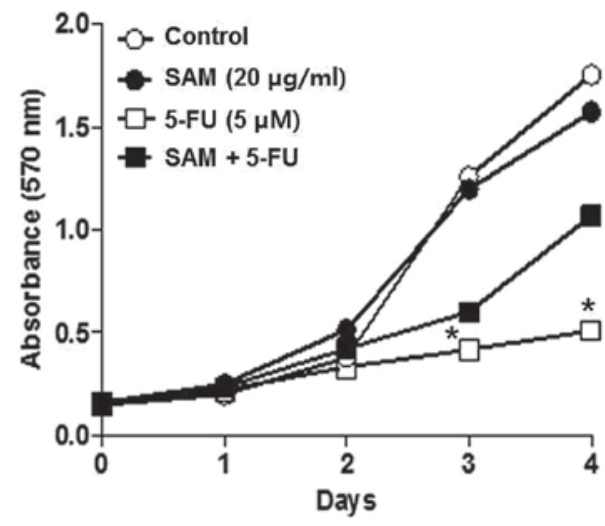

B

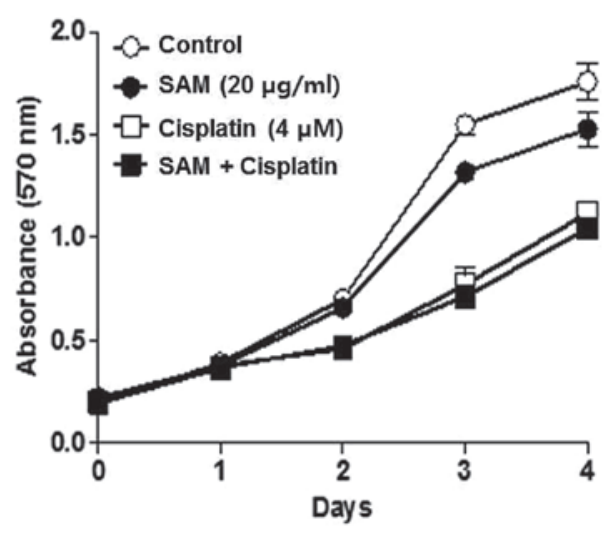

Figure 2. Combination effect of S-adenosyl methionine (SAM) and 5-fluorouracil (5-FU) or SAM and cisplatin. Cells were seeded in 96-well plates, and treated using the indicated drugs for 4 days. Combined treatment of SAM and 5-FU showed a little protection of the 5-FU anticancer effect. This phenomenon was not detected in the combination treatment of SAM and cisplatin. Data are shown as the mean \pm standard deviation of triplicate assays. " $\mathrm{P}<0.05$, compared to the control group.

while the G1 cell population slightly increased, compared with treatment with 5-FU alone. However, combination treatment of SAM and cisplatin resulted in G2/M arrest, similar to cisplatin alone. We quantified data obtained from FACS analysis following treatment with a fixed concentration of SAM $(20 \mu \mathrm{g})$ but with varying concentrations of 5 -FU or cisplatin and the results suggest that a moderate concentration of SAM has a specific protective effect on 5-FU (Fig. 3B and $\mathrm{C}$ ). Therefore, we consider that the protective effect of SAM on the anticancer effect of 5-FU does not require specific cell cycle arrest.

5-FU decreases DNMTs expression but SAM restores the reduction of DNMTs. To analyze which types of cellular methylation are involved in SAM modulation of the anticancer effect of 5-FU, we performed expression analyses on HMTases and DNMTs. The drugs were treated for 3 days and western blot analysis was performed. SUV39H1 or G9a proteins were not detected during SAM treatment or combination treatment with 5-FU, suggesting no induction by SAM or 5-FU (data not shown). However, the expression of DNMT1 and DNMT3A were decreased in 5-FU but not in cisplatin treatment (Fig. 4A). Notably, the combination treatment of SAM and 5-FU restored expression of DNMTs.

Expression of DNMTs was also analyzed using RT-PCR. Consistent with the findings above, 5-FU treatment decreased DNMTs expression in the RNA level but SAM restored the effect of 5-FU on DNMTs expression (Fig. 4B). This result means that protection of SAM on the anticancer effect of 5-FU is exhibited by regulating DNMT expression at the transcriptional level.

\section{Discussion}

Resistance is one of the obstacles to the success of DNA-damaging drug-based chemotherapy. Although the molecular mechanisms of DNA damaging drugs remain controversial, a plausible mechanism is that cellular metabolites 
A
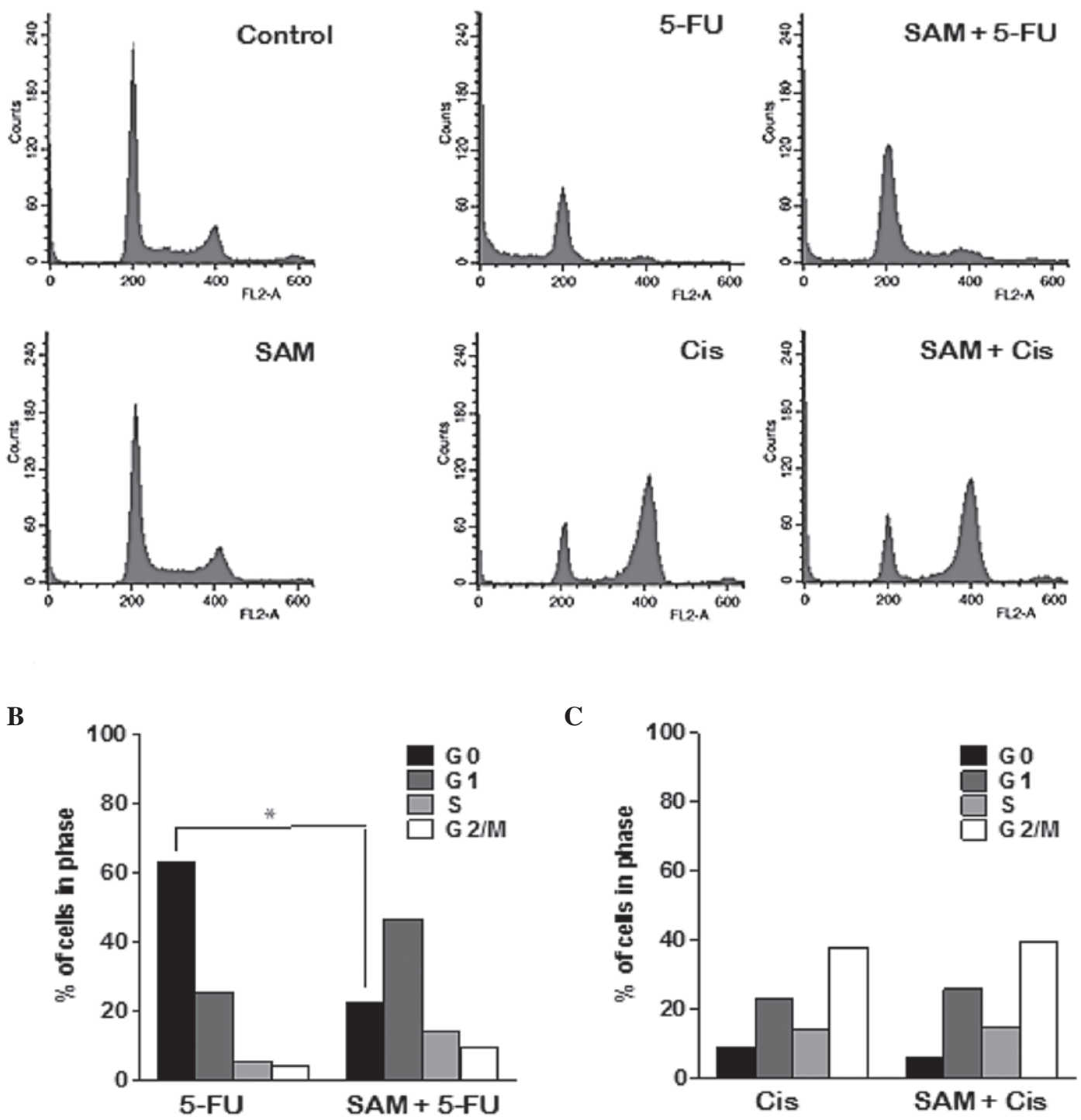

Figure 3. FACS analysis in the combination of 5-fluorouracil (5-FU) or cisplatin with S-adenosyl methionine (SAM). Cells (5x10 cells/well) were seeded and treated with the indicated drugs for $24 \mathrm{~h}$ and fixed with ethanol. Cells were stained with propidium iodide and analyzed with FACScan. 5-FU treatment induced cell death without any relation to a specific cell cycle, but the combined treatment of SAM and 5-FU significantly decreased the dead cell population. The combined treatment of SAM and cisplatin has shown as muh G2/M arrest as with cisplatin alone. "P<0.05, compared to the G0 population treated with 5-FU alone.

A

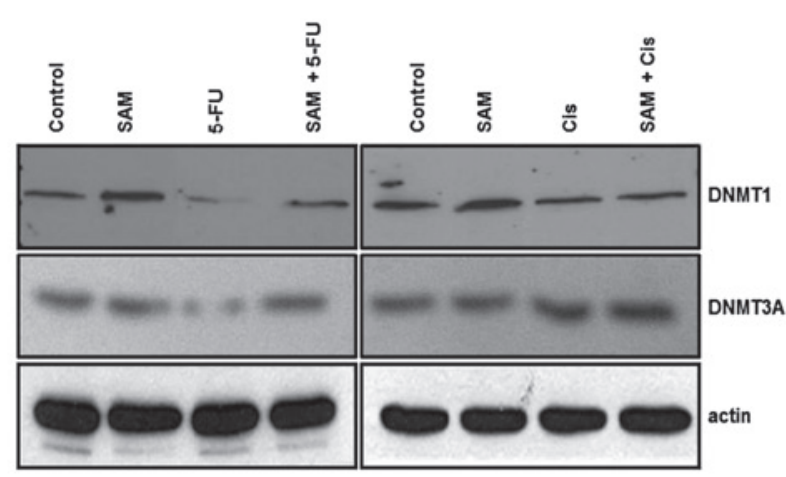

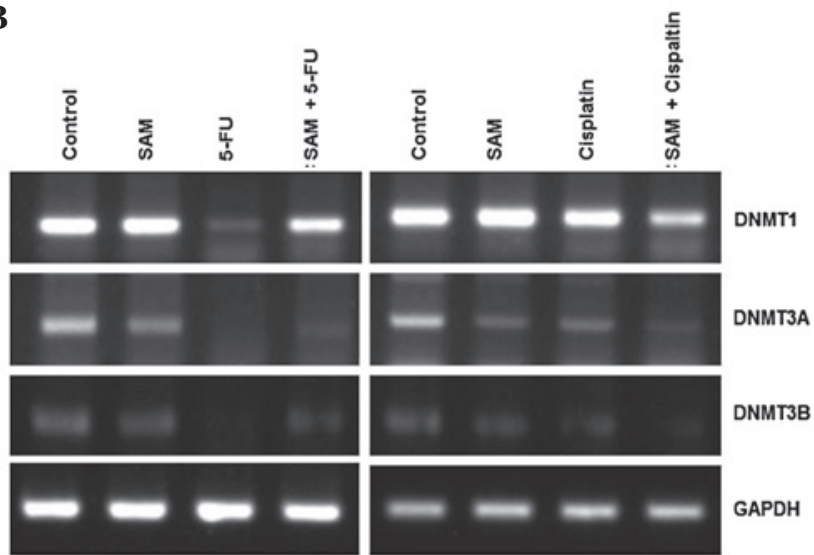

Figure 4. Expression analyses of methylation related proteins in combination of 5-fluorouracil (5-FU) or cisplatin with S-adenosyl methionine (SAM). (A) 5-FU decreased induced DNMT1 and DNMT3A proteins but not in cisplatin. However, combination treatment of SAM and 5-FU restored DNA methyltransferases (DNMTs) protein level. (B) 5-FU treatment decreased most DNMTs expression at the RNA level. SAM restored the effect 5-FU on DNMTs expression. 
regulate cytotoxicity of anticancer drugs. SAM is a physiologic metabolite found in almost every tissue and fluid in the body. Moreover, SAM concentration in the body may also be determined by vitamin B12 and folate (vitamin B6) obtained from dietary sources $(24,25)$. Since use of SAM in a therapeutic setting has not yet been proven when SAM is combined with several anticancer drugs, we examined whether the use of SAM is a regulatory mechanism for the direct modulation of the anticancer effect of DNA-damaging drugs. It has been reported that SAM has inhibitory effects on some carcinoma cells, as well as the proliferation and migration of HUVEC cells at high concentrations $(10,26)$. In our analysis, however, SAM did not exhibit distinct effects on cell growth in A549 lung cancer cells at concentrations ranging up to $40 \mu \mathrm{g} / \mathrm{ml}$. Instead, treatment for a long period of time with SAM demonstrated a small inhibitory effect on the cells. Therefore, the cytotoxic effect of SAM seems to depend on the concentration and treatment time of SAM. Results of the present study have shown that SAM has a protective effect when combined with 5-FU. To achieve this, cytotoxic assays such as MTT assay, cell counting and a viability test were performed. We obtained similar results in that SAM specifically affected the anticancer effect of 5-FU but not for cisplatin. Therefore, we investigated differences between 5-FU and cisplatin to determine this phenomenon. 5-FU treatment induced cell death without specific cell cycle arrest, contrary to cisplatin treatment-nduced G2/M arrest. SAM itself had no specific cell cycle arrest or cytotoxic effect. However, the G1 cell population was increased in the combination of SAM and 5-FU, suggesting that protection of SAM is likely involved in the G1 phase. As a pyrimidine analogue, 5-FU is incorporated in DNA in the $\mathrm{S}$ phase of the cell cycle (27). The anticancer effect of 5-FU occurs in an S phase-active manner, whereas its therapeutic effect is not active when cells are in the G1 phase. As SAM methylates cellular DNA, the anticancer effect of 5-FU is likely reduced during DNA replication. In the case of cisplatin, SAM did not protect the anticancer effect of cisplatin. The reason for this finding is that cisplatin induced G2/M arrest, thus SAM was not able to affect the anticancer effect of cisplatin because cells were already in the G2/M phase.

5-FU treatment was found to regulate a group of cell cyclerelated genes such as cyclin and p53. SAM treatment may regulate gene expression by reversing DNA hypomethylation on gene promoter. As SAM treatment regulated SAM-utilizing genes, we examined the expression of HMTases or DNMTs. 5 -FU or SAM treatment did not produce any induction of SUV39H1 or G9a. Instead, 5-FU treatment decreased most DNMTs expression at the transcriptional level, although SAM had no distinct effect on the expression of DNMTs. However, the presence of SAM restored the effect of 5-FU on DNMTs expression, suggesting that the protective effect of SAM was mediated by the regulation of DNMTs expression. Although cell levels of SAM might affect DNMTs expression, further studies are needed to show the effect of SAM on the expression levels of regulating genes by 5 -FU.

The clinical applications of SAM remain controversial in cancer due to low toxicity. Instead, a growing number of trials have been conducted to find a new anticancer effect of SAM metabolism-related analogues (28-30). Sinefungin, which is a SAM analogue can compete for SAM binding and inhibit the activity of the SAM-dependent methyltransferases. Adenosine dialdehyde inhibits methylation reaction by hindering $\mathrm{SAH}$ hydrolase activity.

SAM, as a methyl donor, plays a versatile regulatory effect in the cells by methylating cell components. The effect of SAM described in the present study may not be universal to the anticancer drugs, but rather specific to 5-FU. Moreover, SAM causes dysregulation of gene expression and cell death by 5 -FU by modulating aberrant DNA methylation. This opens new avenues of how cellular metabolites regulate the anticancer effect of DNA-damaging drugs.

\section{Acknowledgements}

This study was supported by grants from the National Research Foundation of Korea (NRF 2011-0006047) and the Ministry of Education Science and Technology (The Regional Research Universities Program/Medical and Bio-Materials Research Center), Republic of Korea.

\section{References}

1. Dunne AL, Price ME, Mothersill C, et al: Relationship between clonogenic radiosensitivity, radiation-induced apoptosis and DNA damage/repair in human colon cancer cells. Br J Cancer 89: 2277-2283, 2003.

2. Mothersill C and Seymour C: Radiation-induced bystander effects, carcinogenesis and models. Oncogene 22: 7028-7033, 2003.

3. Lu SC and Mato JM: Role of methionine adenosyltransferase and S-adenosylmethionine in alcohol-associated liver cancer. Alcohol 35: 227-234, 2005.

4. Roje S: S-Adenosyl-L-methionine: beyond the universal methyl group donor. Phytochemistry 67: 1686-1698, 2006.

5. Kim KC, Geng L and Huang S: Inactivation of a histone methyltransferase by mutations in human cancers. Cancer Res 63: 7619-7623, 2003 .

6. Fiskus W, Wang Y, Sreekumar A, et al: Combined epigenetic therapy with the histone methyltransferase EZH2 inhibitor 3-deazaneplanocin A and the histone deacetylase inhibitor panobinostat against human AML cells. Blood 114: 2733-2743, 2009.

7. Mato JM, Alvarez L, Ortiz P and Pajares MA: S-adenosylmethionine synthesis: molecular mechanisms and clinical implications. Pharmacol Ther 73: 265-280, 1997.

8. Ji L, Chen Y and Wang Z: Protection of S-adenosyl methionine against the toxicity of clivorine on hepatocytes. Environ Toxicol Pharmacol 26: 331-335, 2008.

9. Wakefield L, Boukouvala S and Sim E: Characterisation of $\mathrm{CpG}$ methylation in the upstream control region of mouse Nat2: evidence for a gene-environment interaction in a polymorphic gene implicated in folate metabolism. Gene 452: 16-21, 2010.

10. Sahin M, Sahin E, Gumuslu S, et al: Inhibition of angiogenesis by S-adenosylmethionine. Biochem Biophys Res Commun 408: 145-148, 2011.

11. Song Z, Zhou Z, Chen T, et al: S-adenosylmethionine (SAMe) protects against acute alcohol induced hepatotoxicity in mice small star, filled. J Nutr Biochem 14: 591-597, 2003.

12. Wang X and Cederbaum AI: S-adenosyl-L-methionine attenuates hepatotoxicity induced by agonistic Jo2 Fas antibody following CYP2E1 induction in mice. J Pharmacol Exp Ther 317: 44-52, 2006.

13. Papakostas GI: Evidence for S-adenosyl-L-methionine (SAM-e) for the treatment of major depressive disorder. J Clin Psychiatry 70: 18-22, 2009.

14. Coppede F: One-carbon metabolism and Alzheimer's disease: focus on epigenetics. Curr Genomics 11: 246-260, 2010.

15. Iacopetta B, Kawakami K and Watanabe T: Predicting clinical outcome of 5-fluorouracil-based chemotherapy for colon cancer patients: is the $\mathrm{CpG}$ island methylator phenotype the 5-fluorouracil-responsive subgroup? Int J Clin Oncol 13: 498-503, 2008.

16. Lombardi L, Gebbia V, Silvestris N, et al: Adjuvant therapy in colon cancer. Oncology 77: 50-56, 2009. 
17. Patel PA: Evolution of 5-fluorouracil-based chemoradiation in the management of rectal cancer. Anticancer Drugs 22: 311-316, 2011.

18. Longley DB, Harkin DP and Johnston PG: 5-fluorouracil: mechanisms of action and clinical strategies. Nat Rev Cancer 3: 330-338, 2003.

19. Giovannetti E, Backus HH, Wouters D, et al: Changes in the status of p53 affect drug sensitivity to thymidylate synthase (TS) inhibitors by altering TS levels. Br J Cancer 96: 769-775, 2007.

20. Kunz C, Focke F, Saito Y, et al: Base excision by thymine DNA glycosylase mediates DNA-directed cytotoxicity of 5-fluorouracil. PLoS Biol 7: e91, 2009.

21. Tominaga T, Iwahashi M, Takifuji K, et al: Combination of p53 codon 72 polymorphism and inactive p53 mutation predicts chemosensitivity to 5 -fluorouracil in colorectal cancer. Int J Cancer 126: 1691-1701, 2010.

22. Bae-Jump VL, Zhou C, Boggess JF and Gehrig PA: Synergistic effect of rapamycin and cisplatin in endometrial cancer cells. Cancer 115: 3887-3896, 2009.

23. Taylor-Harding B, Orsulic S, Karlan BY and Li AJ: Fluvastatin and cisplatin demonstrate synergistic cytotoxicity in epithelial ovarian cancer cells. Gynecol Oncol 119: 549-556, 2010.

24. Taban-Shomal O, Kilter H, Wagner A, et al: The cardiac effects of prolonged vitamin B12 and folate deficiency in rats. Cardiovasc Toxicol 9: 95-102, 2009.
25. Shin W, Yan J, Abratte CM, et al: Choline intake exceeding current dietary recommendations preserves markers of cellular methylation in a genetic subgroup of folate-compromised men. J Nutr 140: 975-980, 2010.

26. Luo J, Li YN, Wang F, et al: S-adenosylmethionine inhibits the growth of cancer cells by reversing the hypomethylation status of c-myc and H-ras in human gastric cancer and colon cancer. Int J Biol Sci 6: 784-795, 2010

27. Robinson HM, Jones R, Walker M, et al: Chk1-dependent slowing of S-phase progression protects DT40 B-lymphoma cells against killing by the nucleoside analogue 5-fluorouracil. Oncogene 25 : 5359-5369, 2006.

28. Hong S, Heo J, Lee S, et al: Methyltransferase-inhibition interferes with neuronal differentiation of P19 embryonal carcinoma cells Biochem Biophys Res Commun 377: 935-940, 2008.

29. Kuratani M, Hirano M, Goto-Ito S, et al: Crystal structure of Methanocaldococcus jannaschii Trm 4 complexed with sinefungin. J Mol Biol 401: 323-333, 2010.

30. Choudhury SR, Balasubramanian S, Chew YC, et al: (-)-Epigallocatechin-3-gallate and DZNep reduce polycomb protein level via a proteasome-dependent mechanism in skin cancer cells. Carcinogenesis 32: 1525-1532, 2011. 